\title{
CONCEPTOS PREVIOS Y CONCEPTOS NUEVOS EN EL ESTUDIO DEL CAMBIO CLIMÁTICO RECIENTE
}

\author{
Javier Martín Vide \\ Grupo de Climatología, Universidad de Barcelona
}

\section{RESUMEN}

Se considera el sistema socioeconómico como el sexto subsistema del sistema climático. Se precisa la definición de cambio climático, que ha de cumplir con claridad la significación estadística en la diferencia entre medias y/o varianzas de al menos una variable climática a escalas temporal y espacial amplias. Se muestra la nueva realidad del calentamiento global reciente de origen antrópico y la muy probable continuación del aumento térmico en las próximas décadas, aunque con incertidumbres, abogándose por la mitigación y la adaptación.

Palabras clave: Adaptación, cambio climático, mitigación, sistema climático, variabilidad.

\section{ABSTRACT}

The author includes the socioeconomic system into the climate system as the sixth subsystem. The climate change concept is defined as a clear statistically significant difference in the average and/or variance of at least one variable on wide temporal and spatial scales. The global warming and its continuation during the next decades is shown, as well as the uncertainties. Finally, mitigation and adaptation are highlighted.

Kew words: Adaptation, climate change, climate system, mitigation, variability.

\section{Introducción}

Para entender lo que es y cabe considerar como un cambio climático hay que tener un buen conocimiento del clima y del sistema climático, lo que exige - es obvio decirlo - análisis rigurosos de los elementos o variables climáticas y atención cuidadosa a la influencia de los factores geográficos y otros. En dos palabras, elementos y factores, cuya definición todo estudiante recordará de sus primeras clases de Climatología. Por una parte, el arsenal de métodos estadísticos y la modelización climática son las 
herramientas más potentes de que disponemos para analizar las variables y las series climáticas y para estimar proyecciones de su evolución futura. Por otra, el conocimiento geográfico es imprescindible para valorar la influencia de los factores geográficos en el clima, especialmente en los países con relieve complejo, como es el caso de España, y también para planificar el territorio y adoptar las medidas de adaptación frente a la nuevas realidades ambientales.

Pero en este asunto, al calor de la actualidad del tema, profesionales de diferentes ramas y aficionados, en algunos casos con expectativas oportunistas, se han subido al carro del tema estrella, atraídos por la importancia del asunto o con el objetivo claro de su beneficio personal, normalmente sin más conocimientos que los del ciudadano de a pie y el input del picoteo de informaciones dispersas en fuentes a veces de credibilidad no garantizada. Es frecuente, por ejemplo, asistir a análisis, valoraciones, proyecciones y atribuciones apresuradas al cambio climático en cuanto ocurre algún episodio meteorológico extremo, que, conviene recordarlo, forman parte de la «normalidad» del clima, o incluso no meteorológico. Por ello, se impone la reflexión pausada, la moderación en las opiniones, sin abandonar, naturalmente, el principio de precaución o cautela ante lo que podemos nominar como una nueva realidad, la del calentamiento global. En esta dirección trata de aportar alguna idea el presente trabajo.

\section{Los conceptos previos}

\subsection{El sistema climático}

Es difícil hablar con conocimiento de causa sobre el clima o, por ejemplo, valorar la importancia de una determinada anomalía climática, de unos años particularmente extremados (secos o lluviosos, cálidos o fríos, etc.), sin tener claros unos cuantos conceptos básicos, los que aquí, a modo de recordatorio, se presentan.

El primer concepto clave es el de sistema climático. Décadas atrás cuando se hablaba del clima, sólo se pensaba en la atmósfera, siendo, sucintamente, su estado estructural, estable en una escala de tiempo algo dilatada (multidecadal, secular, milenaria y aún más), a diferencia del tiempo meteorológico, que es su comportamiento coyuntural, efímero, cambiante, en escalas de tiempo cortas (diaria, por ejemplo). La atmósfera era, pues, el objeto casi exclusivo del análisis del tiempo y del clima. Sin embargo, desde hace algunas décadas ha surgido como nuclear en el estudio del clima presente y futuro el concepto de sistema climático.

El sistema climático es un sistema muy complejo constituido por cinco subsistemas o componentes naturales, entre los cuales existe - por la naturaleza sistémica del conjunto de ellos - numerosos y cuantiosos flujos de energía y materia. Los subsistemas son: la atmósfera; la hidrosfera, sobre todo el océano; la superficie sólida o emergida; la biosfera, fundamentalmente la vegetación; y la criosfera, o capas de hielo y nieve. Los flujos o intercambios de energía, en sus diferentes formas, radiación, convección, etc., y de materia, muy especialmente agua, entre los cinco subsistemas son ingentes. En particular, mediante la evapotranspiración se produce una transferencia fundamental en los balances hídrico y energético del planeta entre las superficies líquida y sólida, y la atmósfera. Con el vapor de agua se cede, no sólo materia hídrica, sino energía, en forma de calor latente desde la superficie a la atmósfera. Los cinco subsistemas funcionan acoplados configurando un todo autorregulado. Alguno de ellos, en especial el océano, presenta una alta inercia en sus comportamientos, como evidencia muy bien la respuesta térmica lenta de sus aguas. 


\subsection{La variabilidad natural}

El segundo concepto clave, sin el cual no puede entenderse lo que constituye un auténtico cambio climático, una anomalía o una tendencia significativa, es el de variabilidad natural del sistema climático. Prácticamente todas las variables climáticas, a cualquier escala temporal que se considere, de minutos a milenios, están continuamente fluctuando, variando con respecto a un valor medio o normal. La temperatura, la dirección y la velocidad del viento, la humedad relativa, etc. muestran continuos pequeños cambios o fluctuaciones; a veces, valores extremos o alejados de su media o de su mediana, en las colas de la distribución de frecuencias; e, incluso, anomalías algo persistentes respecto a los valores de los parámetros de tendencia central. En este sentido, la variabilidad constituye una característica esencial del sistema climático. La propia dinámica interna del sistema, compleja, altamente no lineal, caótica, es fuente de comportamientos variables, al igual que ciertos factores o forzamientos del planeta y exteriores a él. Con relación a este funcionamiento natural variable podemos hablar de variabilidad natural.

¿Y cómo medir la variabilidad natural? La estadística ofrece los llamados parámetros de dispersión, como la desviación tipo, la varianza o el coeficiente de variación, que expresan cuánto se alejan en conjunto los valores de una serie de su media. En particular, el coeficiente de variación - cociente entre la desviación tipo y la media de una serie, a veces expresado en porcentajes - es el parámetro más recomendable para expresar la variabilidad de series climáticas, por ejemplo, de precipitación, cuando, al tiempo, han de compararse. La utilidad del coeficiente de variación en los climas de la Península Ibérica es mayúscula, porque es el mejor índice para discriminar la pluviometría de carácter mediterráneo de la oceánica o marítima templada de latitudes medias. En las series anuales de precipitación, valores ligeramente por encima del $20 \%$ sirven para trazar una frontera continua, que separa bien los dos grupos climáticos citados, con valores inferiores en gran parte de Galicia, Asturias, Cantabria, las provincias vascas litorales y algunos sectores pirenaicos, todos ellos no mediterráneos, de los valores superiores, propios del clima mediterráneo, al sur de la línea citada.

\subsection{El cambio climático}

En algunos casos, a escalas de tiempo en general dilatadas, al menos decadales, y a escalas espaciales globales, hemisféricas o, por lo menos continentales, se producen cambios estadísticos claramente significativos en los valores de los parámetros de tendencia central (media, mediana, etc.) y/o de dispersión (varianza, coeficiente de variación, etc.) de la distribución de frecuencias de alguna variable climática, respecto a un período anterior, en cuyo caso puede hablarse de cambio climático, con efectos destacables sobre los cinco subsistemas naturales del sistema climático. En el caso de la biosfera, por ejemplo, los cambios climáticos suelen comportar modificaciones cuantitativas, existenciales y estructurales, con variación del total de seres vivos, desaparición y especialización o aparición de especies, cambios en los ecosistemas, etc.

Puede considerarse que esta dinámica cambiante «radical», la de los auténticos cambios climáticos, forma parte también de la variabilidad natural del sistema climático, porque sus causas son factores naturales, fundamentalmente cambios en la «constante» solar, o input radiativo solar medio en el límite exterior de la atmósfera en exposición perpendicular a los rayos solares - que, por tanto, no es tan constante como indica su nombre-, por variaciones de la actividad solar (manchas, etc.); ciclos orbitales (excentricidad de la órbita de la Tierra alrededor del Sol, inclinación del eje polar, precesión); mayor o menor actividad 
volcánica, y dinámica y distribución de los continentes. Todo ello, causas naturales, las primeras (resumiendo, el Sol), externas, las segundas (resumiendo, volcanes), endógenas.

Asumido el concepto de variabilidad natural, el investigador o el profano no aceptarán atribuir al cambio climático cualquiera de los innumerables episodios meteorológicos extremos, records climáticos, etc. que se dan a diario en el planeta. Más todavía, un sencillo cálculo de probabilidades evidencia que es muy fácil batir un récord de una variable climática, sin que ello, por tanto, permita afirmar nada sobre un verdadero cambio climático.

Hay que realizar algunas precisiones sobre la definición que encabeza este subapartado. De entrada, lo que ha de cambiar de un modo significativo es el comportamiento estadístico de al menos una variable o elemento climático. Esto se concreta en un cambio estadísticamente significativo del valor de un parámetro de tendencia central, fundamentalmente, la media o la mediana, o del de uno de dispersión, sobre todo la varianza, o su raíz cuadrada la desviación tipo, o el coeficiente de variación, o de ambos tipos de parámetros. Hay que insistir que los parámetros estadísticos comparados pueden ser distintos de la media. En efecto, se asume que un cambio significativo de la media de la temperatura o de otra variable climática, a las escalas temporal y espacial indicadas, cualquiera que sea su causa, constituye un cambio climático, pero también hay que considerar que se produce cuando, sin que la media varíe, cambie significativamente la variabilidad del elemento climático considerado. Por ejemplo, si la temperatura media anual del aire de la superficie del planeta siguiera siendo próxima a $15^{\circ} \mathrm{C}$, como en décadas pasadas, pero los años fueran más contrastados térmicamente, muy fríos y muy cálidos, es decir, constituyendo una muestra estadística con mayor varianza, también habría que hablar de cambio climático. ¿O no notarían tales contrastes las especies vivas y los ecosistemas? Algunas probablemente desaparecerían incapaces de soportar los años más extremos. Igualmente, nuestro sistema socioeconómico padecería las consecuencias.

En segundo lugar, es muy importante tener en cuenta que la simple significación estadística en la diferencia entre los valores de, por ejemplo, dos medias de temperatura o dos varianzas, una de un período anterior y otra de uno actual, es condición necesaria para admitir un cambio climático, pero no suficiente. Hay que pedir que la significación sea nítida, no vale estar cerca del umbral de significación estadística, y, si puede verificarse mediante varios tests estadísticos, hay que hacerlo así. Todos sabemos que la estadística es una herramienta utilísima, imprescindible, pero no debemos olvidar, especialmente el climatólogo, que es manipulable o puede hacerse un mal uso de ella. En el caso de las correlaciones lineales puede llegarse a presuntas relaciones o asociaciones sin ninguna base física, aunque los valores del coeficiente de correlación queden dentro de la significación. $\mathrm{O}$, con otro ejemplo, dependiendo de cómo se agrupen los datos en una distribución de frecuencias, el ajuste a una ley de probabilidad puede verse favorecido o dificultado. El climatólogo ha de rechazar, sin más comentarios, todos los resultados que carezcan de significación estadística. Y los que la tengan sólo delatarán un cambio climático cuando su significación sea alta. Se asume convencionalmente el nivel de significación $\alpha=0,05$, pero mejor, $\alpha=0,01$.

En tercer lugar, asumido lo anterior, es decir, la obligada exigencia de una diferencia con una significación estadística clara, hay que recalcar también que la escala espacial del cambio en la media o la varianza sea - como se indicó anteriormente - global, hemisférica o, al menos, continental, para poder hablar con propiedad de cambio climático. Los cambios a escalas inferiores, sobre todo, los locales, como los urbanos, aun suponiendo variaciones apreciables de la temperatura - la diferencia térmica en el centro de las grandes ciudades con respecto a su periferia, por causa de las islas de calor, supera claramente la elevación de la temperatura media global planetaria-, no tienen un impacto significativo a escalas mayores, no alteran el equilibrio del sistema climático. 
Por último, en cuanto a las causas de la variación significativa de la media y/o de la varianza que exige el cambio climático pueden ser tanto naturales, solares, orbitales, volcánicas, geológicas, etc., algo que ha ocurrido muchas veces en el pasado, como - hay ya que abrir el abanico de posibles causas - de origen antrópico. El aumento de temperatura de las últimas décadas no es nada nuevo en un planeta de 4.500 millones de años; la novedad estriba en su causa - como luego se dirá-, antrópica por primera vez.

En resumen, por cambio climático ha de entenderse una variación con una significación estadística clara en períodos de tiempo algo dilatados, decadales o superiores, en los parámetros de tendencia central y/o de dispersión de al menos una variable climática (temperatura, precipitación, etc.) en el conjunto del planeta o claramente visible en ámbitos continentales o superiores.

\subsection{El efecto invernadero}

La sugerente imagen de la atmósfera como piel protectora del planeta, filtrando las radiaciones interestelares nocivas, es afortunada, pero incompleta. Hay otros gases constituyentes naturales de ella transparentes a la radiación solar, de onda corta, mientras que por el contrario son capaces de absorber la emisión terrestre, que es de onda larga. Tal absorción y su posterior reemisión hacia la superficie hacen que ésta tenga una temperatura apreciablemente superior a la que habría sin la atmósfera. A esto se le llama efecto invernadero, un efecto, por tanto, natural, bueno, que hace el planeta confortable para los humanos. Gracias al efecto invernadero la temperatura media en superficie es la citada de unos $15^{\circ} \mathrm{C}$; sin él es fácil demostrar que sería de $-18^{\circ} \mathrm{C}$, configurando así un planeta menos confortable.

De lo anterior se desprende también que un aumento de los gases de efecto invernadero en la atmósfera ha de comportar un incremento de la temperatura. Y en la actualidad la atmósfera es más rica en gases de efecto invernadero, como el dióxido de carbono $\left(\mathrm{CO}_{2}\right)$, el metano $\left(\mathrm{CH}_{4}\right)$ o el óxido nitroso $\left(\mathrm{N}_{2} \mathrm{O}\right)$, que cien años atrás.

Finalmente, conviene hacer un precisión terminológica, la denominación de efecto invernadero, aunque muy expresiva y fácil de entender, y generalizada, es errónea, porque el aumento de temperatura en el interior de un invernadero se debe fundamentalmente al confinamiento de la convección, o ascenso de aire caliente, por causa del plástico que lo recubre, y no a la absorción de la radiación superficial y su reemisión hacia el suelo.

\section{Los hechos fundamentales sobre la nueva realidad del cambio climático y sus incertidumbres}

\subsection{La principal fuente sobre el cambio climático: El Cuarto IPCC}

El conocimiento sobre el cambio climático nos viene de las investigaciones e informes realizados sobre la evolución pasada, presente y futura de la atmósfera y, en general, del sistema climático. La fuente más amplia y fiable es, sin duda, el IPCC, siglas de Intergovernmental Panel on Climate Change (www.ipcc.ch). Sus informes presentan el funcionamiento y el estado del clima, y su evolución. Recogen, tras un proceso riguroso de revisión, el estado del arte sobre lo anterior a partir de los resultados y conclusiones de publicaciones internacionales de calidad contrastada, y añadiendo a sus afirmaciones un nivel de confianza y de verosimilitud. El Cuarto - el último hasta la fecha - vio la luz en 2007 (IPCC, 2007). Está prevista la presentación del quinto en 2014. La estructura básica del Cuarto IPCC, que ocupa miles de páginas es: 'Base de ciencia física' (primer grupo de 
trabajo), 'Impactos, adaptación y vulnerabilidad' (segundo grupo de trabajo), 'Mitigación' (tercer grupo de trabajo) e 'Informe de síntesis'. En su redacción han participado 450 autores principales y otros 800 en partes específicas, y fue revisado por 2.500 expertos.

Resulta una quimera pretender resumir el amplísimo informe del Cuarto IPCC en las páginas asignadas a este artículo. En todo caso, el autor se ha atrevido a proponer 4 adjetivos, tres de ellos presentes en el documento y otro más añadido, que pueden orientar al lector acerca de lo más sustancial de su contenido (Martín Vide, 2008; Martín Vide y Gallego, 2009). Los cuatro adjetivos aluden al calentamiento global, que se constata que es ya «inequívoco», o sea, cierto, real, auténtico, a la vista de todos los datos disponibles. Cuando, además, se mira hacia el pasado, el calentamiento cabe calificarlo de «inusual», es decir, raro, extraño, por la rápida elevación de la temperatura. Si se busca la causa, puede afirmarse que, con una alta probabilidad, una parte sustancial del calentamiento observado es de origen «antrópico». Finalmente, los escenarios climáticos futuros más probables son, cuando menos, «inquietantes» o preocupantes (no decimos catastróficos), según se deduce de las proyecciones que realizan los modelos climáticos.

\subsection{El aumento de la concentración de gases de efecto invernadero}

En el inicio de 2010 la concentración de dióxido de carbono era de 388 ppm (partes por millón), cuando a finales de la década de los años 50 del siglo XX, en que comienzan las medidas instrumentales, era de 315 ppm (figura 1), y en el último tercio del siglo XIX, con estimaciones, debía de ser de unas 280-290 ppm. En el caso del metano y del óxido nitroso también el tramo final - la última década y el último año - es el que muestra las concentraciones más elevadas.

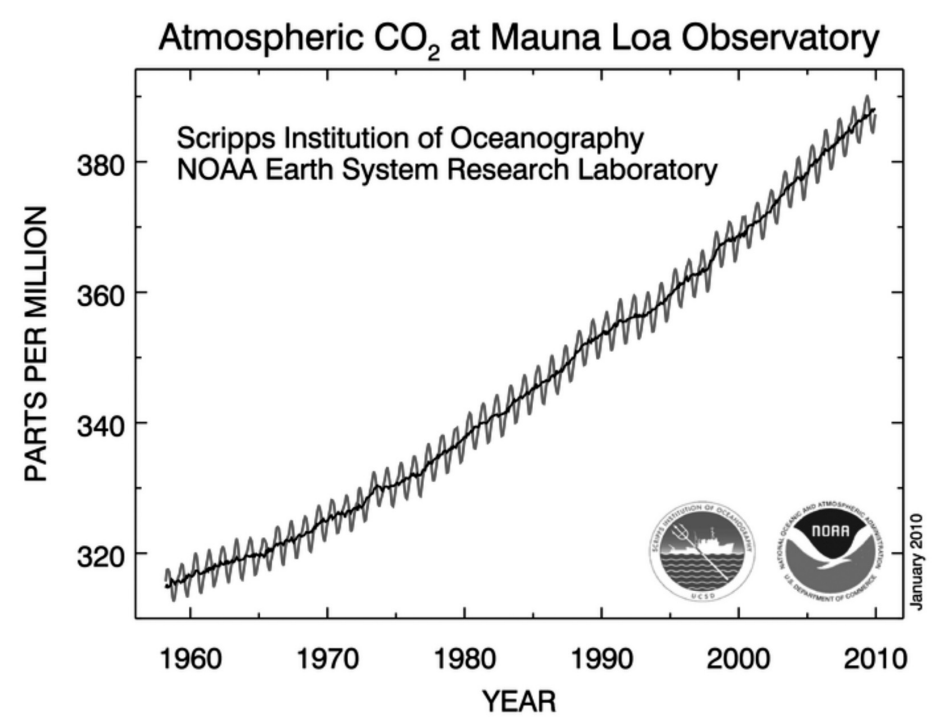

Figura 1: Evolución de la concentración de $\mathrm{CO}_{2}$ en el observatorio de Mauna Loa (Hawaii) desde 1958 hasta 2009.

Fuente: National Oceanic and Atmospheric Administration-NOAA, http://co2now.org/ 
Con lenguaje gráfico, podría decirse que también ha variado la composición química del aire «puro» que respiramos en los ámbitos alejados de las ciudades, aunque ello es totalmente imperceptible para nuestros sentidos.

\subsection{El aumento de la temperatura}

La principal señal del calentamiento global es, precisamente, la elevación de la temperatura media planetaria en superficie. Las series climáticas de temperatura del aire más largas, una vez verificada su calidad y homogeneidad, permiten reconstruir la evolución térmica del planeta desde 1850 (figura 2), en la que sobresale el tramo final, desde la segunda mitad de los años 70 del siglo XX, con un aumento significativo y casi todos los años con una temperatura por encima de la media de referencia. También la Península Ibérica y las Baleares han experimentado un aumento térmico, paralelo al planetario (Castro et al, 2005; Brunet et al., 2006).

La Organización Meteorológica Mundial ha indicado muy recientemente que la primera década del siglo XXI (2000-2009) ha sido la más cálida desde que existe un número apreciable de registros meteorológicos instrumentales (mediados del siglo XIX) y, probablemente, también de los últimos 2.000 años.

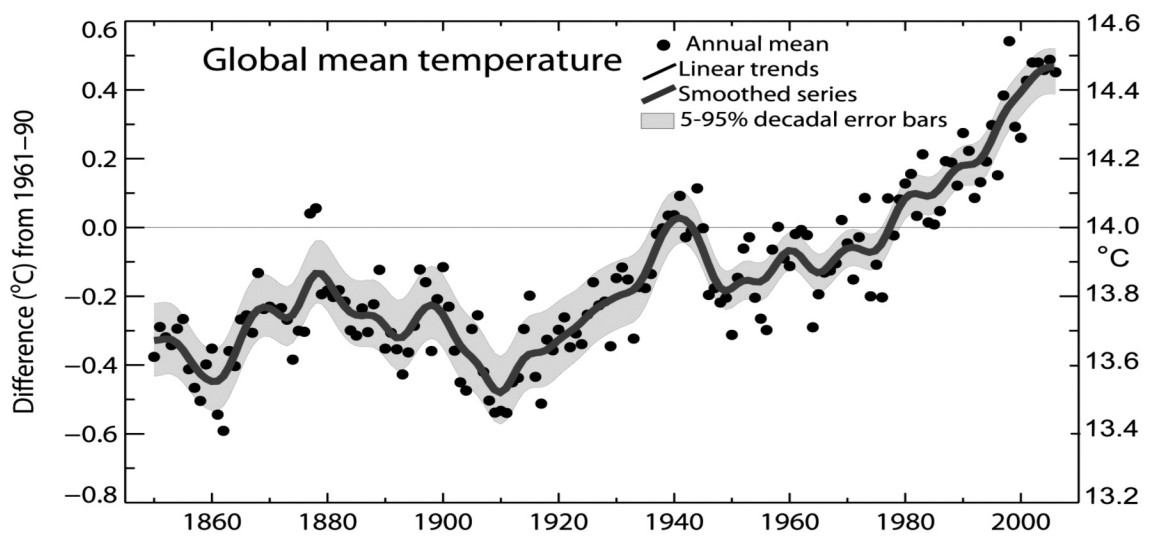

FIGURA 2. Evolución temporal de la temperatura planetaria media anual del aire en superficie en el período 1850-2005.

Fuente: Cuarto IPCC.

\subsection{La subida del nivel marino y el retroceso glaciar}

El segundo hecho de escala global, coherente con el anterior, es la elevación del nivel medio marino. Aunque los registros instrumentales son bastante más escasos que los de la temperatura del aire, y plantean algunas dificultades técnicas, se estima en unos $3 \mathrm{~mm}$ la elevación anual del nivel medio global del mar en la última década analizada por el Cuarto IPCC. Ello es debido no sólo a la fusión del hielo de los glaciares (no a la del hielo marino, que no aumenta el nivel), sino también a la expansión térmica de los océanos, como respuesta a su calentamiento. 
La tercera señal es el retroceso casi generalizado de los glaciares de montaña, que constituyen un testigo inercial y no sometido a alteraciones locales, urbanas o industriales. La mayoría de los glaciares alpinos, andinos, del Himalaya y de otras cordilleras muestran retrocesos muy llamativos en las últimas décadas, que no pueden tener como causa principal más que el calentamiento global. ¿Pueden encontrarse excepciones? Sí, en efecto, en aquellos ámbitos muy fríos en los que haya aumentado la precipitación, sólida, dando lugar a un balance positivo entre la alimentación y la ablación glacial. Esto nos sirve para advertir que los negacionistas a ultranza del calentamiento global siempre encontrarán excepciones, contraejemplos, a favor de su argumento. Excepciones explicables, como la citada, por la complejidad de respuestas y la variedad geográfica y climática del planeta. Debieran reparar en que un muy alto porcentaje de los cambios ocurridos en los sistemas físicos y biológicos en las últimas décadas son coherentes con un mundo más cálido.

\subsection{La causa antrópica del calentamiento reciente}

La realidad del calentamiento global cuenta hoy con un elevado consenso entre la comunidad científica, con pocas voces discrepantes, aunque en los media la proporción y la presencia de negacionistas y escépticos es mayor. No es raro asistir en los platós televisivos y en los estudios de radio al ruego del director del programa de turno, antes de su comienzo, para que los contertulios extremen sus posiciones, lo que ha de permitir el contraste animado de opiniones, expresiones sonoras y un cierto show mediático, todo en aras del mantenimiento de los porcentajes de audiencia. En los últimos años la mayoría de escépticos han aceptado que el planeta es hoy más cálido que 30 años atrás, tal es el volumen y la coherencia de las evidencias en ese sentido. Constatado, pues, el calentamiento del planeta y ya visibles sus señales, queda el asunto polémico en algunos ámbitos negacionistas de la atribución de causas, esto es, si se trata de un cambio o anomalía climática natural o es de origen humano. El perfeccionamiento de los modelos climáticos, que son la herramienta más potente para la comprensión del clima y su evolución, ha dado en los últimos años una prueba robusta para la mayor parte de la comunidad internacional de climatólogos sobre la naturaleza nueva, por su causa, del cambio climático actual. Los modelos climáticos, que son modelos físico-matemáticos tridimensionales de la atmósfera y sus interficies con los restantes componentes del sistema climático, se utilizan para prever el aumento de temperatura que se producirá de aquí a, por ejemplo, 20 ó 50 años, o en 2100. Pero también pueden usarse hacia atrás y, de esta manera, verificar si los valores pronosticados coinciden con los efectivamente medidos en el último siglo. Pues bien, los valores que suministran se ajustan razonablemente a los observados hasta la década de los años 70 del siglo XX sin más que contemplar la variabilidad natural del clima. A partir de esa década los modelos son incapaces de reproducir bien las temperaturas realmente medidas, que quedan sistemáticamente por encima de las pronosticadas. Es decir, las últimas tres décadas tendrían que haber sido apreciablemente más frías, según la influencia de los factores que causan la variabilidad natural, resumidamente radiación solar y actividad volcánica. Sólo cuando en esos modelos se añade la variabilidad de origen antrópico, es decir, fundamentalmente la derivada de los gases de efecto invernadero y de los aerosoles o partículas contaminantes en suspensión, los resultados de los modelos vuelven a ajustarse a los valores observados. Necesitan, por tanto, del factor o «forzamiento» antrópico para dar resultados coincidentes con los ya observados. Se habla en este sentido de la variabilidad antrópica o inducida, que se sobrepone a la natural. Está, por tanto, clara para la mayoría de la comunidad de climatólogos la huella humana, o de las actividades que comportan contaminación atmosférica, en el clima a escala planetaria (Martín Vide et al, 2007, Martín Vide, 2008). 


\subsection{Los escenarios climáticos futuros y sus incertidumbres}

Nadie sabe con exactitud como será el clima futuro, a lo largo del presente siglo y más allá, pero sí que los modelos climáticos permiten dibujar los escenarios climáticos más probables. En cuanto a los efectos y repercusiones futuras del calentamiento global aún las incertidumbres son mayores, por lo que habrá que ser muy comedido en cuanto a las recreaciones del planeta futuro. La tentación de las imágenes catastróficas impostadas sobre los espacios que habitarán nuestros hijos produce un impacto emocional, aparentemente beneficioso para la concienciación del ciudadano, pero al final contraproducente, por fatalista, y el fatalismo sólo conduce a la inacción, y nada científico. Los complejos procesos de retroalimentación del sistema climático han de deparar todavía sorpresas.

Con el conocimiento actual, para el conjunto del planeta las principales previsiones climáticas, a partir de los modelos climáticos, se recogen en el cuadro 1. Ha de tenerse en cuenta que las proyecciones climáticas han de asumir un conjunto de hipótesis socioeconómicas con sus correspondientes niveles de emisiones de gases de efecto invernadero, denominadas escenarios de emisiones. Dependiendo de la cuantía de las emisiones, la elevación prevista de la temperatura será mayor o menor.

\section{Cuadro 1}

PREVISIONES CLIMÁTICAS GLOBALES, SEGÚN LOS MODELOS CLIMÁTICOS, PARA DIFERENTES ESCENARIOS DE EMISIONES

- Aumento de la temperatura media global en superficie para el decenio 2090-2099 de entre 1,8 (escenario B1, emisiones bajas) y $4,0^{\circ} \mathrm{C}$ (escenario A1FI, emisiones altas), respecto a la de las dos últimas décadas del siglo $\mathrm{XX}$.

- Mayor calentamiento en las latitudes altas del hemisferio norte, incluyendo la cuenca del Ártico, y menor en el océano austral.

- Reducción de la superficie y el espesor del hielo marino, pudiendo desaparecer el del Ártico al final de los veranos de las últimas décadas del siglo actual.

- Elevación del nivel medio del mar para el decenio 2090-2099 de entre 0,18 (escenario B1) y 0.59 metros (escenario A1FI), respecto al de las dos últimas décadas del siglo XX.

- Aumento de la precipitación en las latitudes altas y disminución en amplias franjas tropicales y en las regiones mediterráneas.

- Aumento de los riesgos climáticos, sobre todo de las olas de calor y de las precipitaciones torrenciales. En regiones tropicales y subtropicales (mediterráneas), aumento de la frecuencia y la intensidad de las sequías.

- Probable incremento del número de ciclones tropicales (huracanes) más intensos (los de categorías 4 y 5).

Fuente: Cuarto IPCC.

Bajo todos los escenarios plausibles el planeta seguirá calentándose durante las próximas décadas, incluso reduciendo drásticamente las emisiones de gases de efecto invernadero, por, entre otras cosas, la inercia de la componente oceánica del sistema climático. Sobre la precipitación, aunque con más incertidumbres, se pronostican variación al alza en el conjunto del planeta, porque en un planeta más cálido el ciclo del agua se refuerza, al aumentar la 
evaporación y los procesos subsiguientes. Regionalmente, sin embargo, la precipitación puede disminuir, como al parecer sucederá en la cuenca del Mediterráneo. Finalmente, muchos riesgos climáticos, en especial las olas de calor, aumentarán en frecuencia e intensidad, aunque sus efectos dependerán mucho de la vulnerabilidad de la sociedad y de la exposición del territorio (Olcina, 2006).

Para el caso de España las estimaciones futuras más recientes muestran coincidencia en cuanto a la elevación de la temperatura a lo largo del siglo XXI, más marcada en verano y en el interior, $y$, con un apreciable grado de incertidumbre, anuncian una cierta reducción pluviométrica (AEMET, 2009). En todo caso, combinando ambas proyecciones, hay que esperar una reducción de los recursos hídricos. Esto debe estar siempre presente en cualquier planificación hidrológica y en toda actuación de ordenación del territorio en nuestro país.

\section{4. algunos nuevos conceptos}

\subsection{La inclusión del subsistema socieoeconómico en el sistema climático}

Con los datos presentados en el apartado anterior acerca del calentamiento cabe asumir que el sistema socioeconómico forma parte ya, como un subsistema más, del sistema climático, es su sexto componente (figura 3). Esto es así porque la actividad habitual de los casi 6.800 millones de habitantes de la Tierra deja ya huella en los otros susbsistemas a una escala global. El profundo y extenso cambio de usos del suelo ha modificado de una forma sustancial la faz de la tierra, variando de paso albedos, contenidos de agua, topografías, etc., a unas escalas espaciales muy amplias, lo que comporta cambios en los balances radiativos, energéticos e hídricos del planeta en conjunto. La emisión de contaminantes y residuos a los otros subsistemas ha alterado su funcionamiento a escalas que superan con mucho la local. Puede afirmarse ya que los 6.800 millones de humanos producimos un impacto visible en

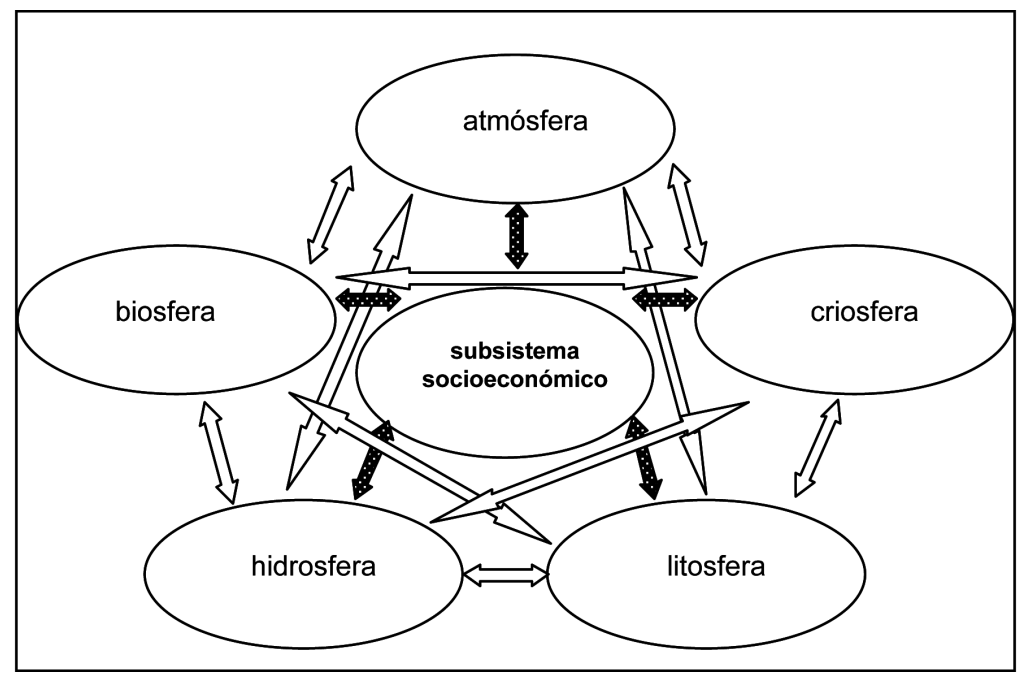

Figura 3. Componentes del sistema climático.

Fuente: Martín Vide (2008). 
el sistema climático considerado en su escala espacial máxima. Y, recíprocamente, nuestro sistema socioeconómico está influido de forma notoria por el comportamiento del medio, de los otros subsistemas, lo que no es una novedad, siempre lo fue así, aunque es plausible comenzar a esperar efectos o impactos más extremados, como respuesta de retorno a la perturbación antrópica.

La consideración del susbsistema socioeconómico como integrante del sistema climático, con impactos notables en los otros subsistemas, conduce inevitablemente a cuestionarse el modelo de desarrollo económico capitalista puro y duro, el modelo energético ineficiente y basado en los combustibles fósiles, la dinámica demográfica explosiva, los desequilibrios sociales y territoriales, la injusticia y la desigualdad entre los humanos, y, en general, el modelo de crecimiento depredador de los recursos y generador de ingentes residuos contaminantes.

\subsection{Calentamiento global, cambio climático y cambio global}

Terminológicamente, los campos semánticos de las expresiones calentamiento global, cambio climático y cambio global son cada uno más amplio que el anterior (figura 4). El cambio climático no sólo comprende la elevación de la temperatura del aire junto a la superficie, y de ésta, sea sólida o líquida, o calentamiento global, que es el hecho más visible constatado, sino otras posibles variaciones en el sistema climático, como, por ejemplo, el aumento o la disminución de la precipitación, según las regiones, o la pérdida de hielo en los glaciares de montaña. A su vez, el concepto de cambio global es más amplio que el de cambio climático, al englobar todos los fenómenos que comportan un cambio sustancial a una escala global, como, además del cambio climático, la pérdida de biodiversidad, el agujero de la capa de ozono, los cambios generalizados de usos del suelo, etc.

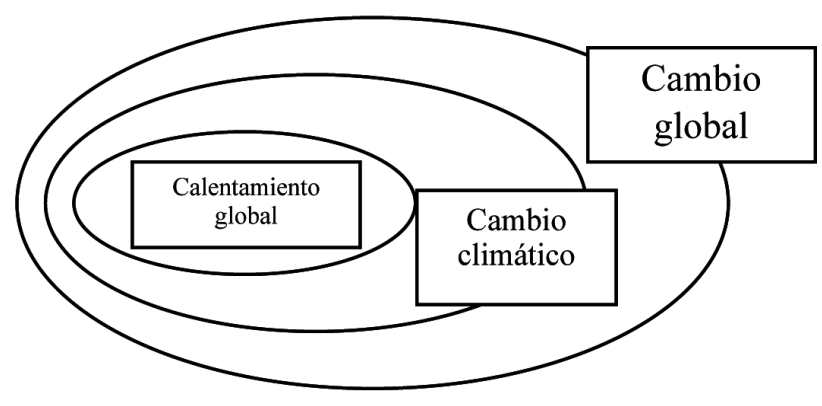

FiguRa 4. Representación esquemática de los campos semánticos de tres expresiones usuales en cambio climático.

\subsection{La mitigación}

Los estudios más recientes sobre proyecciones de la temperatura consideran que continuará aumentando en las próximas décadas, aun reduciendo de forma muy sustancial las emisiones de gases de efecto invernadero, en gran medida por la inercia de los procesos y del comportamiento del sistema climático (Matthews y Caldeira, 2008). Previsión que no debe ser calificada 
de fatalista, sino que ha de servir para la acción responsable, adoptando el compromiso de la mitigación, es decir, de la reducción al máximo de las emisiones de gases de efecto invernadero, sean las producidas por el uso de los combustibles fósiles, sean las derivadas de los usos del suelo, y las de otras fuentes. Se estima que, si el calentamiento sobrepasa unos $2^{\circ} \mathrm{C}$ respecto a la situación previa, podrían producirse algunos procesos irreversibles en el sistema natural, tal como una pérdida generalizada de biodiversidad. La mitigación es, por tanto, en la actualidad uno de los conceptos clave, que comporta un conjunto muy diverso de acciones y buenos hábitos ligados a la eficiencia energética, al empleo de fuentes de energía limpias y renovables, a la mesura y el ahorro en el consumo, y a la sostenibilidad.

\subsection{La adaptación}

Dado que todo apunta a que vamos a tener que vivir en un planeta más cálido, habrá que adaptarse a las nuevas condiciones ambientales, y también económicas y sociales. Adaptación, cuyo significado es el de ajustarse a los nuevos escenarios ambientales reduciendo el efecto de sus peligros e impactos, pero también aprovechando las nuevas oportunidades que ofrecerán. En algunos casos, reducir o minimizar determinados impactos obligará a medidas de adaptación «quirúrgicas», ingenieriles, como la construcción de muros de protección y defensa de nuestras playas, ante la elevación del nivel del mar, o de lo mismo para los márgenes fluviales, al haber sido ocupados inadecuadamente en el pasado, etc. En otros casos se abrirán nuevas oportunidades, como la desestacionalización del turismo en España, al adelantarse el verano en primavera y prolongarse en otoño, por poner un ejemplo próximo, aunque ese beneficio pueda verse contrarrestado por la falta de confort climático en el centro de un verano más caluroso.

\section{Epílogo}

En todo caso, aun si el lector no estuviere convencido de la nueva realidad del cambio climático, o, asumiéndola, le restara importancia, casi seguro que convendrá en la obligada reducción de consumos de bienes, la reutilización de materiales, el reciclaje de lo viejo, etc., lo que se ha venido en denominar sintéticamente como «las erres». Y - añadimostanta o más importancia que las erres citadas tiene la de la reflexión, la buena reflexión y el sentido común, para que la huella humana en el medio físico y en la vida tenga el menor impacto posible.

\section{Agradecimientos}

Al proyecto RECABA (CGL2008-06129-C02-01/CLI, 2009-2011) del Ministerio de Ciencia e Innovación, al Grupo de Climatología (Generalitat de Catalunya) y al Instituto del Agua (Universidad de Barcelona), en los que se inscriben las investigaciones que han dado lugar a las anteriores reflexiones.

\section{Referencias bibliográficas}

AEMET (2009): Generación de escenarios regionalizados de cambio climático para España. Madrid, Ministerio de Medio Ambiente y Medio Rural y Marino.

BRUNET, M.; SALADIE, O.; JONES, P.; SIGRO, J.; AGUILAR, E.; MOBERG, A.; LISTER, D.; WALTHER, A. y LOPEZ, D. (2006): The development of a new dataset of Spanish daily adjusted temperature series (SDATS) (1850-2003), en International Journal of Climatology, ${ }^{\circ}$ 26, pp. 1777-1802. 
CASTRO, M.; MARTÍN-VIDE, J. y ALONSO, S. (2005): «El clima de España: pasado, presente y escenarios del clima para el siglo XXI», en MORENO, J. M. (coord.): Evaluación Preliminar de los Impactos en España por Efecto del Cambio Climático, pp. 1-64, Ministerio de Medio Ambiente, España.

Cuarto IPCC (2007): www.ipcc.ch

MARTÍN VIDE, J. (2008): «La nueva realidad del calentamiento global. Un decálogo del cambio climático», en Scripta Nova, Revista Electrónica de Geografía y Ciencias Sociales. Universidad de Barcelona, $\mathrm{n}^{\circ}$ 12(270):23.

MARTÍN VIDE, J. (coord.); LLEBOT, J. E.; PADILLA, E. y ALCÁNTARA, V. (2007): Aspectos económicos del cambio climático en España. Caixa Catalunya, Barcelona.

MARTÍN VIDE, J. y GALLEGO, J. L. (2009): Apaga la luz. El libro sobre el cambio climático, Davinci Continental, L'Hospitalet de Llobregat.

MATTHEWS, H. D. y CALDEIRA, K. (2008): «Stabilizing climate requires near-zero emissions», en Geophysical Research Letters, ${ }^{\circ}$ 35, L04705.

National Oceanic and Atmospheric Administration-NOAA, ' $\mathrm{CO}_{2}$ now', http://co2now.org/

OLCINA, J. (2006): ¿Riesgos naturales? I. Sequías e inundaciones, Davinci Continental, Mataró. 
